\title{
Thyroid lymphoma: a rare tumor requiring combined
}

\section{management}

\author{
Luca Foppiani ${ }^{1}$, Vincenzo Secondo ${ }^{2}$, Anselmo Arlandini ${ }^{3}$, Paolo Quilici ${ }^{4}$, \\ Manlio Cabria ${ }^{5}$, Patrizia Del Monte ${ }^{1}$
}

${ }^{1}$ Endocrinology, ${ }^{2}$ Internal Medicine, ${ }^{3}$ Surgery and ${ }^{4}$ Pathology, ${ }^{5}$ Nuclear Medicine, Galliera Hospital, Genoa, Italy

\begin{abstract}
Thyroid lymphoma is a rare disease which occurs mainly in elderly females. Most patients with thyroid lymphoma have Chronic Lymphocytic Thyroiditis (CLT), suggesting a role of chronic antigen stimulation in the development of the disease. We present two cases of thyroid Diffuse Large B-cell Lymphoma (DLBCL) diagnosed after surgery (subtotal thyroidectomy) by means of combined histology and immunohistochemistry (positive staining for CD-20) in two elderly female patients presenting at our institution for compressive symptoms (dysphonia in patient 1 , dysphagia in patient 2) due to a gross neck mass. Fine-needle aspiration was compatible with lymphocytic thyroiditis in the first patient and was indeterminate in the second patient. The first patient had a long-lasting history of hypothyroidism due to CLT and was on L-thyroxine replacement therapy, whereas the second patient had normal thyroid function and negative thyroid autoantibodies. After surgery both patients underwent chemotherapy (cyclophosphamide, doxorubicin, vincristine and prednisone (CHOP) plus rituximab). At one-year follow-up both patients were disease-free. Thyroid lymphoma is an uncommon tumor which requires prompt diagnosis and combined management for a high rate of cure to be achieved.
\end{abstract}

Key words: Chemotherapy, Immunohistochemistry, Surgery, Thyroid lymphoma

\section{INTRODUCTION}

Primary thyroid non-Hodgkin lymphomas (NHL), mostly Diffuse Large B-cell Lymphoma (DLBCL), account for $1-5 \%$ of thyroid tumors and $1-2.5 \%$ of all lymphomas. ${ }^{1-3}$ NHL has its highest prevalence in females in the sixth to seventh decade of life..$^{1-3}$ Most of these tumors originate from B lymphocytes

Address for correspondence:

L. Foppiani, M.D., Ph.D., Endocrinology, Galliera Hospital, Mura delle Cappuccine 14, 16128 Genova,

Fax: 0039105634306, E-mail: luca.foppiani@galliera.it

Received 04-05-09, Revised 25-05-09, Accepted 10-06-09 and rarely from T cells. Pathologically, two thirds of thyroid NHL are represented by DLBCL, arising either de novo or in the context of a concomitant indolent NHL of the mucosa-associated lymphatic tissue (MALT). ${ }^{4}$ From a pathophysiological point of view, chronic antigen stimulation is involved in the pathogenesis of NHL and virtually all thyroid NHL derive from antigen-experienced B-cells, harbouring immunoglobulin heavy chain variable region (IGHV) genes extensively targeted by the somatic hypermutation (SHM) process. Up to two thirds of thyroid NHL harbour mutation of genes, namely CD95/FAS, expressed in the germinal centre. ${ }^{4}$ From 
a clinical point of view, more than onehalf of patients with thyroid NHL have biochemical/ultrasonographic features of Chronic Lymphocytic Thyroiditis (CLT) (nearly $50 \%$ with hypothyroidism) ${ }^{1}$. The relative risk of primary thyroid lymphoma is 60-70 times higher in patients with CLT than in the normal population and the usual time of development of thyroid lymphoma after the onset of CLT is about 20-30 years. ${ }^{1,5,6}$ The current management of this rare disease involves surgery, chemotherapy with classic and new drugs and radiotherapy. We here describe the clinical, morphological and histological features of a thyroid lymphoma which developed in two elderly women with quite different characteristics, and the difficulties in establishing the diagnosis.

\section{CASE REPORTS}

CASE 1: In 1995 a 70-year-old-woman was referred to our unit following the diagnosis of severe hypothyroidism: TSH: $38.3 \mathrm{mIU} / \mathrm{L}$ (NV: 0.3-4.5), FT4: $6.4 \mathrm{pmol} / \mathrm{L}$ (NV: 10.3-25.7), during evaluation for mental impairment. The patient's face was mixedematous and the thyroid gland was enlarged. Thyroid autoantibodies were increased and ultrasonography (USG) showed a multinodular goitre. Fine-needle Aspiration Biopsy (FNAB) of the prevalent (15 mm) left lobe hypoechogenic nodule was compatible with lymphocytic thyroiditis. L-thyroxine therapy up to $100 \mu \mathrm{g} /$ day normalized FT4 and TSH over time and yearly controls were scheduled. Over the years, thyroid morphology did not change significantly; marked hypoecogenicity and multiple small unmodified hypoechogenic nodules. In November 2007, FNAB on the prevalent enlarged $(30 \mathrm{~mm})$ left lobe thyroid nodule detected by a further USG confirmed the previous diagnosis of lymphocytic thyroiditis. Since the volume of all other thyroid nodules was below 1 $\mathrm{cm}$, no FNAB was performed. Two months later, dysphonia occurred and phoniatric examination revealed left vocal cord palsy. Thyroid USG showed a large hypoechogenic mass (maximum diameter $4.5 \mathrm{~cm}$ ) arising from the left lobe invading the isthmus, and dislocating the trachea. Subtotal thyroidectomy was performed. At surgery, the neoplasia infiltrated the loco-regional muscles and the oesophagus. Combined histological and immunohistochemical analyses led to the diagnosis of DLBCL with a high proliferation index (Ki-67: 75\%) and positive CD20, CD10 and BCL6 (Figure 1). The patient underwent age-tailored chemotherapy (cyclophosphamide, doxorubicin, vincristine and prednisone (CHOP), 4 cycles every 4 weeks) plus rituximab (anti CD-20 antibody, 6 cycles every 4 weeks) (R-CHOP). Mild chemotherapy-related toxicity was observed: grade I WHO for hemoglobin, grade 2-3 WHO for leukocyte count. At 6- and 12month follow-up, total-body CT showed no relapse of the disease.

CASE 2: A 65-year-old woman complained of a large palpable swelling in the neck with associated dysphagia of recent onset. Neck USG revealed a gross $(7.5 \times 4 \times 4 \mathrm{~cm})$ hypoechogenic mass, which originated from the thyroid isthmus and invaded both lobes. Thyroid function was normal (TSH: $2.5 \mathrm{mIU} / \mathrm{L}$ ) and thyroid autoantibodies were negative. FNAB of the mass disclosed thyrocytes with oxyphil cytoplasm and lymphocytes (THY3). Subtotal thyroidectomy was performed. At surgery, the neoplasia infiltrated the loco-regional muscles and the trachea. Integrated histological and immunohistochemical analyses led to the diagnosis of DLBCL with a high proliferation index (Ki-67: 60\%) and positive CD20, BCL2 and BCL6 (Figure 1). Post-surgical hypothyroidism was satisfactorily managed by replacement therapy with L-thyroxine (up to $75 \mu \mathrm{g} /$ day). CHOP (4 cycles every 4 weeks) plus rituximab (6 cycles every 4 weeks) chemotherapy (R-CHOP) with mild hematological side-effects (anemia, leukopenia) was applied. At 6- and 12-month follow-up, total-body CT did not show relapse of the disease.

\section{DISCUSSION}

Thyroid lymphoma is an uncommon tumor which occurs primarily in middle-aged to older aged females. DLBCL is the most frequent (50-60\%) histologic subtype of thyroid lymphomas. ${ }^{1-6}$ Epidemiological evidence and indirect molecular findings from immunoglobulin heavy chain variable region (IGHV) gene studies suggest that chronic persistent antigen stimulation leads to malignant transformation and possibly constitutes a significant pathogenetic mechanism of thyroid NHL. Nearly half of thyroid NHL has a clinical history of CLT and up to two thirds of cases show pathological clues consistent with CLT. Moreo- 

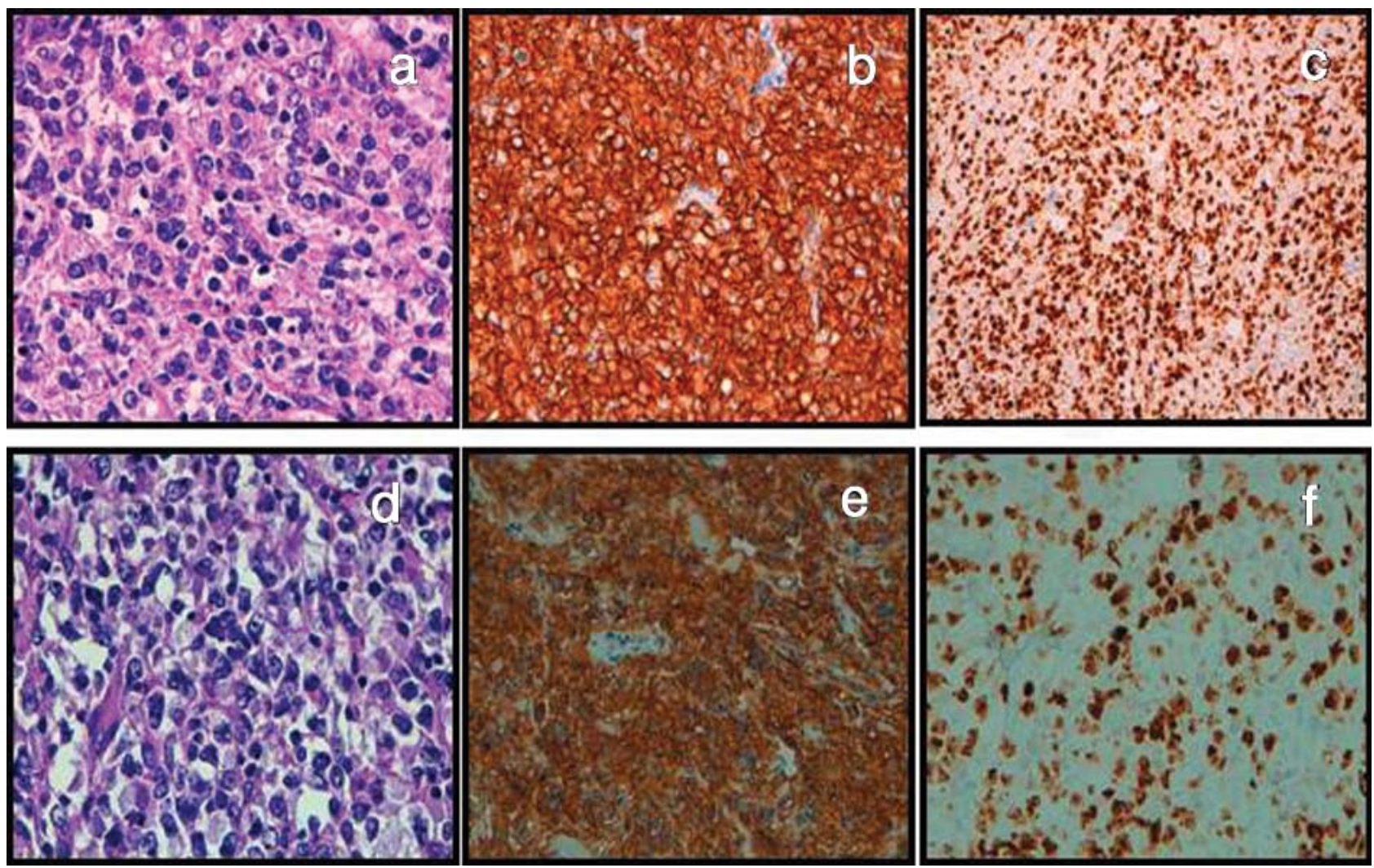

Figure 1. Representative images of the thyroid diffuse large B-cell lymphoma: histology (Haematoxilin-eosin), positivity for CD-20, and Ki-67 staining in patient 1 (a [x400], b [x400], c [x100] and patient 2 (d [x630], e [x400], f [x400]).

ver, clonal IGVH rearrangements carried by thyroid NHL may already be evident among the oligoclonal rearrangements which characterize the preceding phase of CLT and a fraction of thyroid NHL use the same IGHV genes utilized by antithyroid autoantibodies. ${ }^{4,7}$ Current molecular data provide evidence on the oncogenesis of thyroid lymphoma (TL). Thus aberrant activity of somatic hypermutation (ASHM) is regarded as a mechanism contributing to the development of lymphoid malignancies, since it may introduce activating mutations and may cause genetic instability, favouring chromosomal translocation. This could play a role in the development of follicular lymphoma (FL) but possibly not in lymphoma of marginal zone B-cell type (MZBCL). DLBCL includes ASHM negative and positive cases indicating heterogeneity in pathogenetic mechanisms involved. ${ }^{8}$ As regards the relationship between CLT and thyroid NHL, recent findings suggest a putative scenario in which SHM misfires multiple proto-oncogenes within a tissue microenvironment prone to NHDL development, such as the CLT microenvironment. In this context, aberrant SHM would represent a very early event in the process of B-cell clonal transformation. . $^{4-8}$

Most patients with primary thyroid lymphoma have a rapidly growing painless thyroid mass causing compressive symptoms such as hoarseness, dysphagia and dyspnea. ${ }^{1,6,9-11}$ These features overlap with that of the more life-threatening anaplastic thyroid carcinoma (ATC). The appearance of thyroid lymphoma on USG is that of a well-defined hypoecogenic mass, although this feature is not unique. ${ }^{11}$ Since most thyroid lymphomas develop in the thyroid gland with pre-existing CLT, the presence of lymphocytes on cytology may be erroneously attributed to thyroiditis; the differential diagnosis with ATC can also be difficult. ${ }^{5}$ Overall, the results of FNAB for the diagnosis of thyroid lymphoma are often inconsistent and its diagnostic accuracy varies widely $(25-80 \%) .,{ }^{5,10}$ For these reasons, cytology is not deemed the most accurate tool for the diagnosis of thyroid lymphoma. Tissue biopsy (core 
or incisional) is usually necessary in order to make a definitive diagnosis of thyroid lymphoma using combined histology and immunohistochemistry. ${ }^{1,5,9}$

Our two patients showed a common clinical presentation (a recent-onset of painless, large neck mass causing compressive symptoms) but several different biochemical features (hypothyroidism and CLT in the first patient, euthyroidism and negative thyroid autoantibodies in the second). The appearance on USG, of a large hypoechogenic mass was similar in both patients. The presence of compressive symptoms with negative (patients 1) and indeterminate (patient 2) cytology, made surgery a useful tool for both the relief of symptoms and for a definitive histological diagnosis. Indeed, combined histology and immunohistochemistry revealed in both patients a DLBCL with positivity of CD20 and a high proliferation index. Only in the first patient the lymphoma originated from the germinal centre (CD10+ and $\mathrm{Bcl} 6+)$. In both patients $\mathrm{CHOP}$ plus rituximab (R-CHOP) chemotherapy achieved a clinical response. This therapy is the gold standard for this type of lymphoma and is generally well tolerated; it must, however, be tailored to each patient. While CHOP regimen has already been used for many years in the treatment of DLBCL with significant results in progression-free survival and overall survival, the introduction in recent years of rituximab, monoclonal antibody against the membrane antigen CD-20 has improved the long-term prognosis and treatment of patients with DLBCL at clinically high risk. ${ }^{3}$ Today six cycles of R-CHOP chemotherapy without radiation are an effective option for patients who have no bulk disease. Radiotherapy is another useful tool in selected cases, but has a long latency period and short- and long-term toxicity and can exacerbate obstructive symptoms due to tissue swelling. Previous literature reports on patients with DLCL treated with chemotherapy/surgery/radiotherapy alone or in combination showed a complete remission in $40-70 \%$ of patients; $;^{1,5,10,12}$ by contrast, thyroid mucosal associated lymphoid tissue (MALT) lymphoma showed significantly higher complete response. ${ }^{5}$ Finally, Matsuzuka et al reported several years ago a $100 \%$ survival rate at 8 years in a subset of 16 patients with thyroid lymphoma treated with radiation therapy combined with CHOP chemotherapy. ${ }^{11}$ The surgical management of thyroid lymphoma has changed over time. Nowadays, most authors believe that total thyroidectomy unnecessarily exposes patients to the risk of such surgery (recurrent laryngeal nerve damage and hypoparathyroidism) without conferring any survival advantage. ${ }^{13}$ Currently, a larger percentage of patients undergo surgery in order to achieve precise histological diagnosis rather than therapeutic benefit. Surgery, however, still plays an important role in the management of patients with severe airways obstruction or compressive symptoms. ${ }^{14}$ Overall, with appropriate therapy thyroid NHL has a rather favourable course; however its prognosis depends on histology, local spread, and the stage of the disease at diagnosis. A combined management is mandatory in order to achieve a high percentage of cure.

Although the length of follow-up is limited to one year which precludes definite conclusions on the outcome of therapy applied, it is nevertheless indicative of a favourable evolution. The history of the two cases underlines the difficulties in establishing cytological confirmation of the diagnosis. Hence, a high index of suspicion should lead to tissue biopsy with the application of histology and immunohistochemistry for early diagnosis and appropriate therapy.

\section{REFERENCES}

1. Thieblemont C, Mayer A, Dumontet C, et al, 2002 Primary thyroid lymphoma is a heterogeneous disease. J Clin Endocrinol Metab 87: 105-11.

2. Swerdlow SH, Campo E, Harris NL, et al, 2008 The WHO Classification of Tumors of Haematopoietic and Lymphoid Tissues, p 233-237, IARC, Lyon 2008.

3. Friedeberg J, Fisher R, 2008 Diffuse large B-cell lymphoma. Hematol Oncol Clin N Am 22: 941-952.

4. Rossi D, 2009 Thyroid lymphoma: beyond antigen stimulation. Leuk Res 33: 607-609.

5. Hwang Y-C, Kim TY, Shong YK, et al, 2009 Clinical characteristics of primary thyroid lymphoma in Koreans. Endocr J Feb 18 [Epub ahead of print].

6. Widder S, Pasieka JL, 2004 Primary thyroid lymphoma. Curr Treat Opt Oncol 5: 307-313.

7. Moshynska OV, Saena A, 2008 Clonal relationship between Hashimoto thyroiditis and thyroid lymphoma. J Clin Pathol 61: 438-444.

8. Takakuwa T, Miyauchi A, Aozasa K, 2009 Aberrant somatic hypermutation in thyroid lymphomas Leuk Res 33: 649-654

9. Chiofalo MG, Corazelli G, Franco R, De Chiara A, Pezzullo L, 2008 Thyroid lymphoma: early clinical suspicion may be critical for cure. J Endocrinol Invest 
31: 739-40.

10. Ha CS, Shadle KM, Medeiros LJ, et al, 2001 Localized non-Hodgkin lymphoma involving the thyroid gland. Cancer 91: 629-635.

11. Matsuzuka F, Miyauchi A, Katayama S, et al, 1993 Clinical aspects of primary thyroid lymphoma: diagnosis and treatment based on our experience of 119 cases. Thyroid 3: 93-99.

12. Colovic M, Matic S, Kryeziu E, Tomin D, Colovic N,
Atkinson HD, 2007 Outcomes of primary thyroid nonHodgkin's lymphoma: a series of nine consecutive patients. Med Oncol 24: 203-208.

13. Ruggiero FP, Frauenhoffer E, Stack BC Jr, 2005 Thyroid lymphoma: a single institutions' eperience. Otolaryngol Head Neck Surg 133: 888-896.

14. Rochow-Meyer GY, Sywak MS, Reeve TS, Delbridge LW, Sidhu SB, 2008 Surgical trends in the management of thyroid lymphoma. EJSO 34: 576-580. 